damage to the autonomic and sensory ganglion neurons leading to clinical manifestation of AASN.

T IRIOKA

$M$ YAMADA

$M$ YAMAWAKI

Y SAITO

H MIZUSAWA

Department of Neurology and Neurological Science, Graduate School of Medicine, Tokyo Medical and Dental University, 1-5-45 Yushima Bunkyo-ku, Tokyo 113-8519, fapan

M YAMADA

Department of Neurology,

Kanazawa University School of Medicine, fapan

H MIURA

Department of Internal Medicine, Social Insurance Chuo General Hospital, Japan

Correspondence to: Dr T Irioka

irioka@tc4.so-net.ne.jp

1 Colan RV, Snead OC, Oh SJ, et al. Acute autonomic and sensory neuropathy. Ann Neurol 1980;8:441-4.

2 Yasuda T, Sobue G, Mokuno K, et al. Clinicopathophysiological features of acute autonomic
and sensory neuropathy: a long-term follow-up and sensory neuropathy: a long-tern

3 Quattrini A, Comi G, Nemni R, et al. Axonal neuropathy associated with interferon- $\alpha$ treatment for hepatitis C: HLA-DR immunoreactivity in Schwann cells. Acta Neuropathol 1997, 94:504-8.

4 Adachi H, Mukai E, Okuda S, et al. A severe case of acute autonomic and sensory neuropathy. Clinical Neurology (Tokyo) 1998;38: 663-8. (In Japanese.)

5 Pavesi G, Gemignani F, Macaluso GM, et al. Acute sensory and autonomic neuropathy: possible association with Coxsackie B virus infection. I Neurol Neurosurg Psychiatry 1992; 55:613-15.

Neuropathic pain with vesical and rectal hyperreflexia and cocontraction after pelvic surgery

Pelvic and pudendal nerve injury can occur during extirpative visceral surgery such as radical hysterectomy. ${ }^{12}$ Many of these patients develop severe chronic pelvic pain and bladder symptoms, and are often referred to neurologists with suspicion of lumbosacral plexus lesions or disc disease. There are few or no signs on examination, and patients are often considered to be "hysterical", despite having severe symptoms. Here, we describe two patients in whom severe pelvic pain and bladder dysfunction developed after hysterectomy, and who demonstrated detrusor and rectal hyperreflexia with cocontractions, features usually associated with lesions of the CNS. Whereas spinal cord sensitisation is well recognised after somatic nerve injury, our studies provide the first clear evidence for its development after visceral nerve injury in humans, and a method for its detection using ambulatory urorectodynamics.

Patient 1, a 42 year old woman, was diagnosed as having carcinoma of the cervix 5 years previously and underwent Wertheim's hysterectomy, followed by chemotherapy and pelvic irradiation. She developed severe persistent vaginal pain and hypersensitivity, which prevented her from having sexual intercourse, and subsequently bladder dysfunction, which required intermittent selfcatheterisation. She received several analgesic drugs without benefit. Neurological and pelvic examination and spinal imaging, were normal. An ambulatory urorectodynamics study (after prior written informed consent) showed vesical instability associated with unstable urethral function; simultaneous abnormal rectal contractions and an associated fall in anal pressure were also recorded (figure). A repeat ambulatory study confirmed these findings, which showed clear temporal correlation with her symptoms.

Patient 2, a 69 year old woman, had had vaginal hysterectomy for prolapse with bladder repair 23 years previously. She developed severe persistent pelvic pain, exacerbated during micturition. She reported that her vagina and rectum felt continuously stretched, "as if sitting on a baby's head", and found it difficult to sit without discomfort. She developed progressive urinary and rectal urgency. Neurological examination and anal tone were normal. Vaginal examination showed exquisite tenderness on the left. Nerve conduction study showed prolonged pudendal nerve latency (left $2.7 \mathrm{~ms}$, right $2.4 \mathrm{~ms}$; normal range $2.0 \pm$ $0.2 \mathrm{~ms}$ ).

Magnetic resonance imaging of the spine and pelvis were normal, as was flexible cystoscopy. An ambulatory urorectodynamic study (after prior written informed consent) showed urethral instability, unstable vesical contractions with simultaneous abnormal rectal contractions, and falls in anal pressure.

These patients had severe symptoms but no clinical signs, and abnormalities were detected only after pudendal nerve conduction and urorectodynamic studies, which disclosed visceral hyperreflexia in both cases. Patient 1 probably had injury to the pelvic nerves, which is well recognised after extensive hysterectomy. ${ }^{1}$ Patient 2 had pudendal nerve damage, supported by the nerve conduction study. Our patients did not have neurological signs suggestive of CNS lesions, but demonstrated features usually associated with such lesions, namely detrusor and rectal hyperreflexia. Visceral hyperreflexia can occur after spinal cord lesions and in the absence of obvious neurogenic lesions but its occurrence after peripheral nerve damage is not well recognised. ${ }^{34}$

The likely explanation for visceral hyperreflexia in our patients is increased barrage originating from injured sensory nerves, leading to spinal cord sensitisation; this mechanism is well established after somatic peripheral nerve injury, but rarely considered after pelvic surgery. ${ }^{5}$ Persistent visceral noxious input to the spinal cord could lead to reflex activation of the intermediolateral cell column, ${ }^{6}$ the increased output of which may in turn increase bladder and rectal contractions. Other relevant evidence of spinal cord disinhibition in our patients is the loss of the normal inhibition

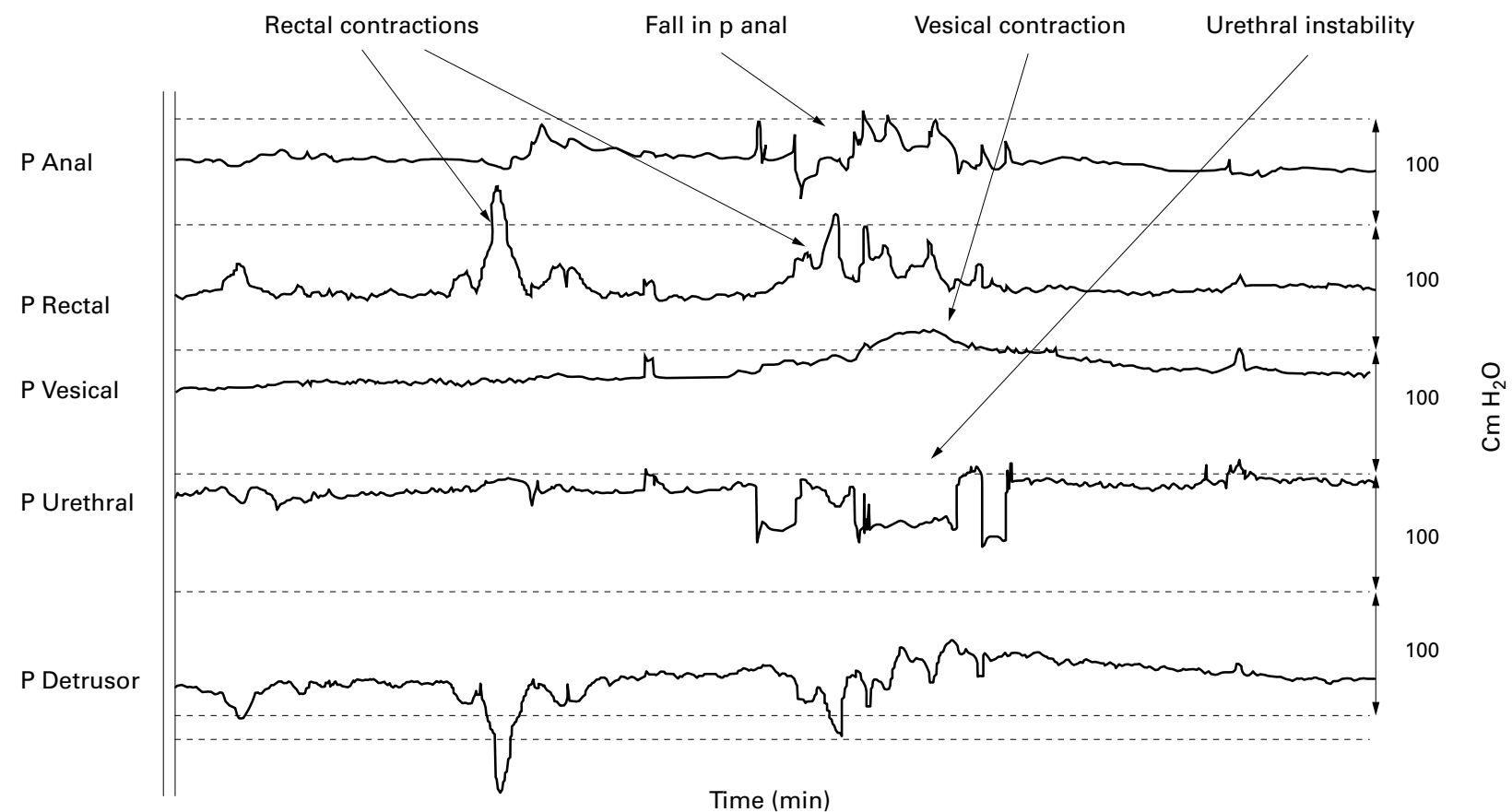

Figure 1 Eight minute trace from first ambulatory study on patient 1, showing uninhibited vesical contraction, urethral instability, and abnormal rectal contraction associated with a fall in anal pressure. Note simultaneous vesical and rectal contractions (cocontractions). 
of urinary bladder contraction induced by rectal and vaginal stimulation ${ }^{7}$ and the development of bladder and rectum cocontractions, which have not been reported previously.

Our cases show how pelvic surgery could be complicated by persistent neuropathic pain and bladder and bowel hypersensitivity, and further studies of spinal cord excitability are needed to clarify underlying mechanisms. Early recognition and initiation of analgesic treatment for neuropathic pain isessential to prevent the condition becoming intractable.

\section{P SHEMBALKAR}

P ANAND

Peripheral Neuropathy Unit, Department of Neurology, Imperial College School of Medicine, Area A, Ground Floor, Hammersmith Hospital, Du Cane Road, London, W12 ONN, UK

I JUNAID C FOWLER

Department of Urology, The Royal London Hospital, Whitechapel, London E1 $1 \mathrm{BB}, \mathrm{UK}$

N S WILLIAMS

Academic Department of Surgery, The Royal London Hospital, Whitechapel, London E1 1BB, UK

Correspondence to: Professor P Anand

P.Anand@ic.ac.uk

1 Shingleton HM, Thompson JD. Cancer of the cervix. In: Rock JA, Thompson JD, eds. Linde's operative gynaecology. Philadelphia: Lippincot Raven, 1997:1413-99.

2 Benson JT, McClellan E. The effect of vaginal dissection on the pudendal nerve. Obstet Gynecol 1993;82:387-9.

3 Fowler CJ. Neurological disorders of micturition and their treatment. Brain 1999;122. tion and

4 Lowe E, Anand P, Terenghi G, et al. Increased NGF levels in the urinary bladder of women NGF levels in the urinary bladder of women
with idiopathic sensory urgency and interstitial with idiopathic sensory urgency and
cystitis. Br 7 Urol 1997;79:572-7.

5 Zermann DH, Ishigooka M, Doggweiler R, et al. Postoperative chronic pain and bladder dysfunction: windup and neuronal plasticity: do we need a more neurourological approach in pelvic surgery ? F Urol 1998;160 102-5.

6 Anand P, Gibson SJ, McGregor GP, et al. A VIP containing system concentrated in the lumbosacral region of human spinal cord. Natur 1983;305:143-5.

7 Morrison JFB. The neural control of the bladder. In: Bloom SR, Polak JM, Lindenlaub
E, eds. Systemic role of regulatory peptides: sympoE, eds. Systemic role of regulatory peptides: sympoSium, Oosterbeek, Netherlands, 2-6 May 1982.

\section{Peripheral nerve ischaemia after internal iliac artery ligation}

Ligation of the internal iliac (hypogastric) arteries has been used to control serious obstetric and pelvic bleeding. It is generally well tolerated in the young obstetric or gynaecological patient, presumably because of an extensive collateral blood supply. ${ }^{1-3}$ Acute lumbosacral plexopathies have been described, however, in older patients with vascular disease when the internal iliac arteries are interrupted. ${ }^{4-8}$ We report on a teenage patient with similar peripheral nerve ischaemia after bilateral internal iliac artery ligation for postpartum haemorrhage.

An 18 year old woman presented at 40 weeks gestation with mildly raised blood pressures, trace proteinuria, oliguria, and generalised oedema. She was diagnosed with pre-eclampsia and admitted for induction. When induction was unsuccessful, she underwent a caesarean section, which was complicated by uterine atony and a postpartum haemorrhage with an estimated blood loss of
$2500 \mathrm{ml}$. After bilateral uterine artery ligation failed to control the bleeding, bilateral internal iliac artery ligation was performed with resultant haemostasis.

On the first postoperative day, she complained of left buttock pain and difficulty moving her left leg. Superficial skin breakdown over the sacrum and buttocks was noted on the second postoperative day. She developed a fever and fundal tenderness on day 4. Helical CT of the abdomen and pelvis disclosed residual gas and fluid within the endometrial canal consistent with endometritis, which was treated with intravenous antibiotics. No retroperitoneal haematoma was present. Neurological evaluation on the fifth postoperative day was limited by pain but disclosed normal strength, sensation, and reflexes in the arms and the right leg. Strength in the left leg was 2 to $3 / 5$ on hip flexion and knee extension and 3 to $4 / 5$ on ankle plantarflexion, ankle dorsiflexion, and toe extension. Sensation was diminished to all modalities in the entire left leg below the hip. The left patellar and ankle stretch reflexes were absent.

Magnetic resonance imaging of the thoracolumbar spinal cord was unremarkable. An initial magnetic resonance angiogram (MRA) of the pelvis showed segmental occlusions of both internal iliac arteries with distal reconstitution greater on the right than on the left. The left superior gluteal artery was not visualised. Revascularisation was considered but deferred due to the concomitant active pelvic infection. Peripheral pulses remained strong, and Doppler ultrasound of both legs showed no evidence of distal thrombus.

Nerve conduction studies 1 week after ligation were extremely limited and difficult to interpret due to generalised oedema. Sural and peroneal sensory responses were absent bilaterally. Right peroneal and left tibial motor responses were normal. A small left peroneal motor response was present in the anterior tibilais muscle. Electromyography was not performed at that time.

The fevers and endometritis gradually cleared, and over the next month left leg strength improved slowly, but incompletely, with greater proximal (4-5 in hip flexion and knee extension) than distal (2-3 in ankle plantarflexion, 0 in ankle dorsiflexion) recovery. The left leg continued to have diminished sensation to all modalities and remained areflexic. The superficial skin necrosis progressed to an open non-healing ulcer $7 \mathrm{~cm} \times 5 \mathrm{~cm}$ over the sacrum and left gluteal musculature. Magnetic resonance imaging of the region disclosed additional tissue necrosis subcutaneously along the left posterolateral buttock and inflammation in the surrounding subcutaneous tissues and underlying gluteal musculature with extension into the left sacroiliac joint. There was no evidence of rectal, uterine, or bladder ischaemia.

A follow up MRA of the pelvis 6 weeks after ligation demonstrated persistent segmental occlusion of both internal iliac arteries and less collateral flow on the left compared with the right. Again, the superior gluteal artery was not visualised on the left but appeared to fill on the right.

Electromyography of selected muscles of the left leg at that time ( 6 weeks after ligation) showed $2+$ to $4+$ fibrillations and positive sharp waves in the vastus lateralis, tibialis anterior, and lateral gastrocnemius muscles, consistent with acute denervation. There were no voluntary units in the tibialis anterior and low firing rates in the gastrocnemius. Low amplitude polyphasic motor units in vastus lateralis suggested early proximal recovery. Nerve conduction studies showed diminished left sural sensory amplitudes and slowed velocities $(2.8 \mu \mathrm{V}, 36.0 \mathrm{~m} / \mathrm{s})$. The left peroneal motor responses were markedly attenuated, and the left posterior tibial motor velocities were slowed $(32.0 \mathrm{~m} / \mathrm{s})$. The right sural sensory $(13.2 \mu \mathrm{V}, 42.0 \mathrm{~m} / \mathrm{s})$ and peroneal motor $(2.3 \mathrm{mV}, 46.0 \mathrm{~m} / \mathrm{s})$ responses were normal.

In general, the internal iliac artery divides into an anterior and a posterior division. The anterior division is formed by the inferior gluteal artery and its branches, which supply the pelvic viscera, the lower buttocks, and the back of the thigh. The posterior division is formed by the superior gluteal artery and its branches, which supply the gluteal musculature, the femoral nerve, and the sciatic nerve roots. ${ }^{9}$

Ligation of the internal iliac arteries has been accepted as a safe and effective means of controlling serious haemorrhaging from the uterus or lower pelvis after delivery or after gynaecological surgery. ${ }^{1-3}$ The lack of ischaemic complications from ligation of the internal iliac artery is thought to be due to the multiple sources of collateral blood flow present in the pelvis.

There are, however, reports of buttock ischaemia or lumbosacral plexopathies as a complication of interruption of the internal iliac arteries during aortic bypass procedures or aortoiliac aneurysm resection..$^{4-5}$ In a study of 11 patients (mean age 67, range 53 to 87 ) with aortoiliac occlusive disease or aortoiliac aneurysmal disease, seven developed ischaemic injury to the lumbosacral plexus after bilateral internal iliac artery ligation. ${ }^{6}$ In four of those patients, buttock necrosis with extension to the bony pelvis was also seen. In another report, four women (mean age 37, range 33 to 47 ) with insulin dependent diabetes and end stage renal disease developed ipsilateral lumbosacral plexopathies when the internal iliac artery was ligated during kidney transplantation. ${ }^{7}$ Electromyography showed denervation of the tibialis anterior, gastrocnemius, and vastus medialis in one patient and of the tibialis anterior and gastrocnemius in another. Ischaemia of the sciatic and femoral nerves and buttocks also occurred after internal iliac artery embolisation in patients with terminal pelvic malignancies who received radiotherapy. ${ }^{8}$

Our 18 year old patient developed a combination of leg weakness, leg numbness, and buttock necrosis after internal iliac artery ligation, as described in older vasculopathic patients. In the patients described in the literature, as in our patient, the clinical and electromyographic findings do not distinguish between combined femoral and sciatic nerve lesions, a lumbosacral plexopathy, or a combination of the two. Our patient's presentation, however, can be most succinctly explained by an infarction in the territory of the left superior gluteal artery and its branches, resulting in ischaemia to the gluteal musculature, the femoral nerve proper, and the sciatic nerve roots. This localisation is supported by serial MR angiograms of the pelvis in which the left superior gluteal artery and its branches were not visualised.

It has been shown that, in experimental ligation of the internal iliac artery of rats, moderate ischaemia is associated with demy- 